\title{
Spectrophotometric Determination of a Thiobarbituric Acid-Reactive Substance in Human Hair
}

\author{
Jenn-Yuan Sheu,* Po-Hua Chen,** Wei-Chang Tseng,*** Chang-Yu Chen,*** Li-Yu TsaI,** \\ and Yeou-Lih HuANG*ं
}

\author{
*Graduate Institute of Medicine, Kaohsiung Medical University, Kaohsiung, Taiwan \\ **School of Technology for Medical Sciences, Kaohsiung Medical University, Kaohsiung, Taiwan \\ ***Department of Medical Technology, Fooyin University, Kaohsiung, Taiwan
}

\begin{abstract}
A simple and sensitive spectrophotometric method for the determination of a thiobarbituric acid-reactive substance (TBARS) in human hair has been developed. The proposed method is based on the formation of a red-colored product by the reaction of products of lipid peroxidation with thiobarbituric acid in an acidic medium. The absorbance of the resulting red product was measured at $534 \mathrm{~nm}$. The linear dynamic range was between 1.0 and $20 \mu \mathrm{mol} / \mathrm{L}$. The recoveries were $98.3-105.0 \%$, and the relative standard deviations (RSD) were $0.32-1.24$, respectively. TBARS in digested hair sample was stable for 3 days at room temperature. It was found that, using this method, the hair TBARS concentration in smokers $(0.116 \pm 0.030 \mu \mathrm{mol} / \mathrm{g}, n=30)$ was significantly higher than that in non-smokers $(0.096 \pm 0.015$ $\mu \mathrm{mol} / \mathrm{g}, n=30)(p<0.05)$.
\end{abstract}

(Received November 11, 2002; Accepted March 3, 2003)

\section{Introduction}

Lipid peroxidation, the oxidative breakdown of polyunsaturated fatty acids, is widely accepted as a general mechanism for cellular injury and death. ${ }^{1}$ Lipid peroxidation has been implicated in diverse pathological conditions, including atherosclerosis, aging and cancer, and in toxicity associated with certain solvents, drugs and metals. ${ }^{2}$ A variety of analytical methods are available for evaluating lipid peroxidation, include the following techniques: iodometry of peroxides in oils and fats $;^{3}$ fluorometry of lipofuscin-like substances in serum ${ }^{4}$ and lipid hydroperoxides in foodstuffs; ${ }^{5}$ spectrophotometry of conjugated dienes in lipids ${ }^{6}$ and of peroxides in olive oil ${ }^{7}$ chemiluminescence detection of lipid peroxides in dairy products $^{8}$ and in human plasma; ${ }^{9}$ gas chromatography of ethane or pentane in exhaled breath; ${ }^{10}$ malondialdehyde evaluation with or without a thiobarbituric acid (TBA) reaction and capillary electrophoresis, ${ }^{11}$ and high-performance liquid ${ }^{12}$ or capillary gas chromatographies. ${ }^{13}$

The thiobarbituric acid reaction, quantified by colorimetry or fluorimetry, is the most widely used method for studying lipid peroxidation in both animals and humans with disorders. ${ }^{3}$ In addition, because of its simplicity, the TBA method is a useful and easy screening technique for evaluating lipid peroxidation in biological systems. ${ }^{14}$ Therefore, in the biomedical research area, the assays for thiobarbituric acid-reactive substances (TBARS) are much more widely used than any other index of lipid peroxidation. ${ }^{3}$ However, no available method has been established for the detection of TBARS in hair.

As far as sampling and storage are concerned, the non-

$\dagger$ To whom correspondence should be addressed.

E-mail: yelihu@kmu.edu.tw invasive sampling, the ease with which it can be collected, its stability during storage, and its high concentrations of most analytes, as compared with other body tissues and fluids, all make hair a suitable specimen for biological monitoring studies and potentially for diagnostic considerations. ${ }^{15}$ Furthermore, hair analysis can be applied to any population group. Hair analysis has been shown to be a useful and important diagnostic tool for detecting the consumption of illicit or therapeutic drugs. ${ }^{16,17}$ Because each hair follicle is surrounded by a system of capillary vessels at the root, the same information as present in blood is found in hair. ${ }^{18}$ Moreover, there is increasing evidence that hair can be considered a minor excretory organ, supporting its use as a biopsy material representing the body. ${ }^{19}$ Hair has been successfully used to trap nutrients, trace elements, alcohol metabolites, and drugs of abuse for latter release and measurement by standard techniques. ${ }^{19-22}$

Mogos et al. demonstrated that the cholesterol and lipid contents in hair could be used as a long-term retrospective evaluation of the lipid metabolism on a single hair specimen. ${ }^{23}$ Auwerter et al. reported that the fatty acid ethyl esters hair concentrations can be used as markers for excessive alcohol consumption with relatively high accuracy. ${ }^{24}$ Hirano et al. showed the direct analysis of human hair for a vanadium determination. $^{25}$ As far as lipid peroxidation is concerned, numerous reports have dealt with its measurement in various kinds of biological samples. ${ }^{3}$ However, to our knowledge, lipid peroxidation in hair has not been reported. Therefore, in the current study a sensitive and simple spectrophotometric method is described for the determination of TBARS in human hair. The proposed method was then applied to measure TBARS in the hair of smokers and non-smokers. 


\section{Experimental}

\section{Principle}

Under an acidic condition and elevated temperature, the products of lipid peroxidation were reacted with TBA to form a red complex possessing an absorption maximum at $534 \mathrm{~nm} .{ }^{3}$ A red pigment was extracted into butanol and measured by spectrophotometry. The TBA reaction was standardized by the analysis of tetraethoxypropane standard solutions. The analytical results were expressed as micromole TEP per gram of hair.

\section{Apparatus}

A Hitachi U 2000 (Tokyo, Japan) double-beam UV/VIS spectrophotometer was used for electronic spectral measurements. The $\mathrm{pH}$ measurements were made with a Radiometer (Model PHM 210, Lyon, France) $\mathrm{pH}$ meter in combination with a calomel glass electrode.

\section{Reagents and solutions}

All reagents used were of analytical reagent grade. Deionized water was used to prepare all solutions. Working standard solutions were prepared from a stock standard solution of 1,1,3,3-tetraethoxypropane (Sigma, St. Louis, MO, USA); the contents were diluted to the mark with ethanol solution $(40 \%$, $\mathrm{v} / \mathrm{v})$. Phosphoric acid solution was prepared by diluting a suitable volume of $85 \% \quad \mathrm{H}_{3} \mathrm{PO}_{4}$ reagent (w/v) (Merck, Darmstadt, Germany) to one liter with distilled water. Thiobarbituric acid was prepared by dissolving certain amounts of thiobarbituric acid reagent (Sigma, St. Louis, USA) with gentle heating in $500 \mathrm{ml}$ of distilled water. Butylated hydroxytoluene (BHT) was purchased from Sigma (St. Louis, MO, USA). Butanol was purchased from Merck (Darmstadt, Germany).

\section{Sample preparation}

To develop the method, a pool of hair clips from random individuals was collected from a barbershop. In order to remove external any contamination and sebum, all hair samples were thoroughly washed before analysis with acetone and distilled water, and dried at room temperature. Because the pooled hair was a heterogeneous sample, we made it into a composite by mixing and remixing randomly in a glass bottle. About $40 \mathrm{mg}$ of the washed hair sample was cut into smaller pieces and weighed. The hair was digested on a heating block at $80^{\circ} \mathrm{C}$ with $2 \mathrm{ml}$ of $2 \mathrm{M} \mathrm{NaOH}$ for $20 \mathrm{~min}$. The digested hair sample was then centrifuged for $10 \mathrm{~min}\left(3500 \mathrm{~g}, 25^{\circ} \mathrm{C}\right)$ to remove residues.

\section{Color development and spectrophotometric detection}

Triplicate aliquots of each supernatant of digested hair sample $(0.5 \mathrm{ml})$ were pipetted into Pyrex tubes $(13 \mathrm{~mm}$ diameter, 150 mm length) containing $1.5 \mathrm{ml}$ of a $\mathrm{H}_{3} \mathrm{PO}_{4}(9 \%$ w/v) solution. The reagent blank was prepared by pipetting $0.5 \mathrm{ml}$ of an ethanol solution into a Pyrex tube containing $1.5 \mathrm{ml}$ of an $\mathrm{H}_{3} \mathrm{PO}_{4}(9 \%$, w/v) solution. Standard samples were prepared by pipetting $0.5 \mathrm{ml}$ of each TEP working standard solution $(2.5,5$, 10, $20 \mu \mathrm{mol} / \mathrm{l}$ ) into Pyrex tubes containing $1.5 \mathrm{ml}$ of a cold $\mathrm{H}_{3} \mathrm{PO}_{4}(9 \%$, w/v) solution. A $0.5 \mathrm{~mL}$ portion of a thiobarbituric acid $(30 \mathrm{mM})$ solution was added to each tube, and the contents were mixed with a vortex mixer. The tubes were capped with marbles and placed for $60 \mathrm{~min}$ in a boiling water bath. The tubes were cooled to room temperature in a basin containing tap water. Butanol $(2.5 \mathrm{~mL})$ was added to each tube, and the

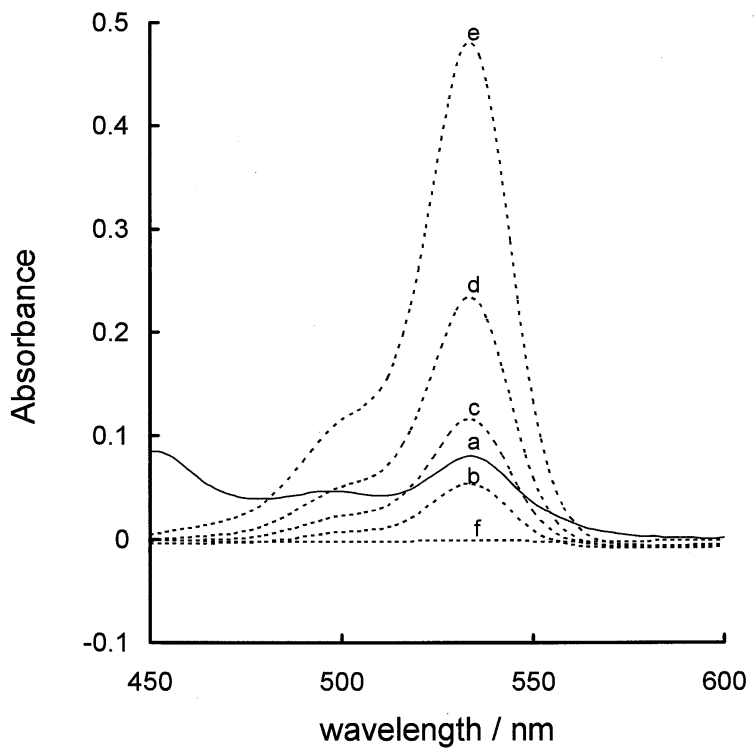

Fig. 1 Absorption spectra of (a) $0.5 \mathrm{ml}$ of hair sample, $1.5 \mathrm{ml} 9 \%$ (w/v) $\mathrm{H}_{3} \mathrm{PO}_{4}, 0.5 \mathrm{ml} 30 \mathrm{mM}$ TBA. (b) - (e) $0.5 \mathrm{ml}$ of TEP $(2.5,5.0$, 10.0 , and $20.0 \mu \mathrm{M}$, respectively), $1.5 \mathrm{ml} 9 \%(\mathrm{w} / \mathrm{v}) \mathrm{H}_{3} \mathrm{PO}_{4}, 0.5 \mathrm{ml} 30$ mM TBA. (f) Reagent blank containing $1.5 \mathrm{ml} 9 \%$ (w/v) $\mathrm{H}_{3} \mathrm{PO}_{4}, 0.5$ $\mathrm{ml} 30 \mathrm{mM}$ TBA.

contents were mixed for $20 \mathrm{~s}$ with a vortex mixer. The contents of each tube were transferred to a conical polystyrene centrifuge tube (13 mL volume), capped, and centrifuged for $20 \mathrm{~min}$ $\left(1000 \mathrm{~g}, 25^{\circ} \mathrm{C}\right)$. Each butanol extract was transferred with a Pasteur pipet into a spectrophotometer cuvet (1 cm light path) in the sample cell. Spectrophotometric scans were performed from 400 to $560 \mathrm{~nm}$, with butanol in the reference cell. The TBARS were determined at the maximum absorption $(534 \mathrm{~nm})$.

\section{Statistical analysis}

All data are presented as means \pm SD. Data were statistically analyzed using the Student's t-test, with P-values less than 0.05 as the level of statistical significance.

\section{Results and Discussion}

\section{Absorption spectra}

The absorption spectrum of the TBARS in butanol was studied over the wavelength range of 400 to $560 \mathrm{~nm}$ (Fig. 1). The absorption spectrum of the complex is a asymmetric curve with the maximum absorbance at $534 \mathrm{~nm}$. The reagent did not show any absorbance at this wavelength. Therefore, a wavelength of $534 \mathrm{~nm}$ was used in all absorbance measurements. The molar absorptivity of the complex calculated from the absorbance value was found to be $2.58 \times 10^{4}$ $1 \mathrm{~mol}^{-1} \mathrm{~cm}^{-1}$ at $534 \mathrm{~nm}$.

\section{Effect of the $\mathrm{H}_{3} \mathrm{PO}_{4}$ concentration}

The effect of the $\mathrm{H}_{3} \mathrm{PO}_{4}$ concentration on the absorbance of TBARS was studied using the recommended procedure. Upon varying the $\mathrm{H}_{3} \mathrm{PO}_{4}$ concentration between 9 to $15 \%(\mathrm{w} / \mathrm{v})$, the absorbance of the TBARS remained constant (Fig. 2). Hence, $9 \%(\mathrm{w} / \mathrm{v}) \mathrm{H}_{3} \mathrm{PO}_{4}$ was used for subsequent studies.

\section{Effect of the TBA concentration}

Figure 3 illustrates the effect of the TBA concentration on the absorbance of TBARS. Varying the TBA concentration in the 


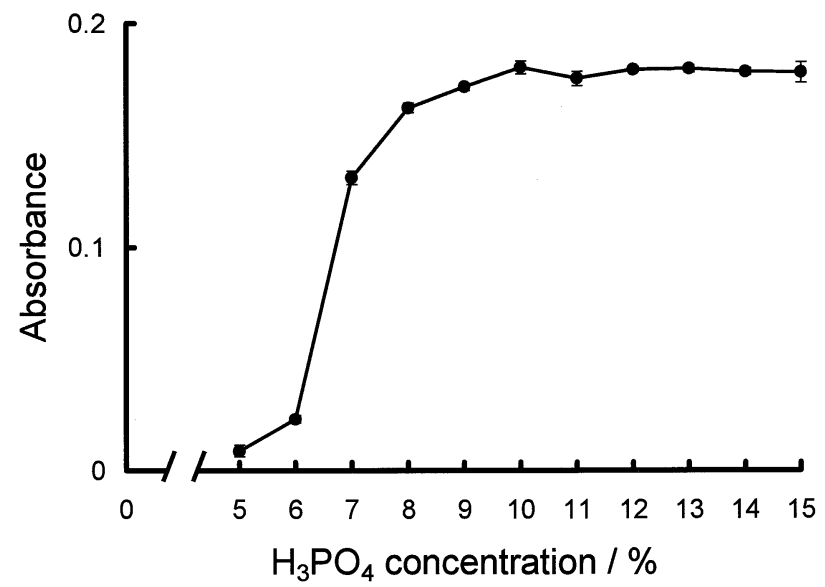

Fig. 2 Effect of the $\mathrm{H}_{3} \mathrm{PO}_{4}$ concentration on the absorbance of TBARS in a hair sample. The values represent the mean \pm standard deviation of $n=3$.

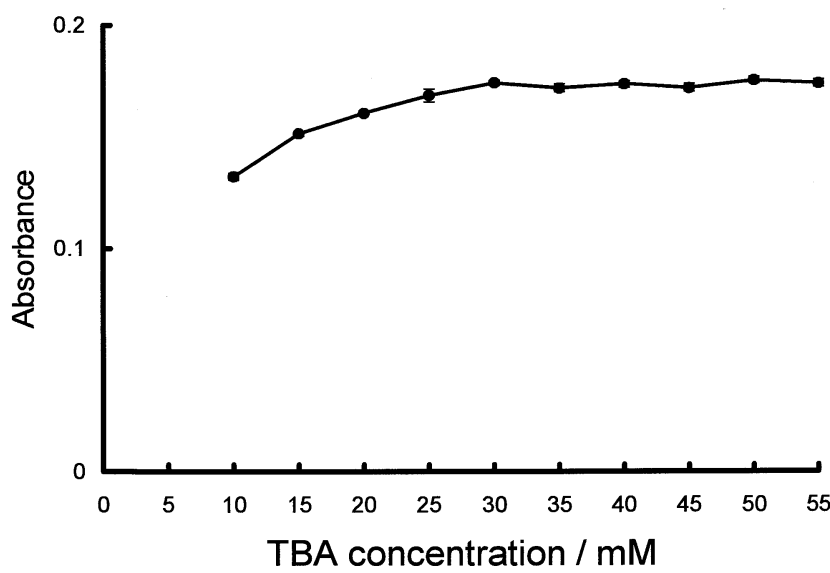

Fig. 3 Effect of the TBA concentration on the absorbance of TBARS in a hair sample. The values represent the mean \pm standard deviation of $n=3$.

reaction mixture from 10 to $30 \mathrm{mM}$ gradually increased the yield of TBARS from the hair samples. Nevertheless, increasing the TBA levels further did not show any additional effect. Therefore, a TBA level of $30 \mathrm{mM}$ was selected for routine TBARS measurements.

\section{Effect of BHT concentration}

In general, high temperature and low $\mathrm{pH}$ during the reaction may cause an artificial formation of products of lipid peroxidation. ${ }^{26}$ Some antioxidants, such as the naturally occurring tocopherols and synthetic BHT, can be used to reduce the artificial formation of lipid peroxidation products. ${ }^{26}$ In this work, to examine the effect of BHT on the formation of artificial lipid peroxidation products, various levels of BHT were added to hair samples before a reaction. The results indicated that no clear change in the absorbance of the TBARS was found upon adding different BHT concentrations ( 0 to $1.0 \%)$ to the hair samples before a reaction.

\section{Analytical performance}

Under the optimized conditions mentioned above, the calibration graph is linear for $1-20 \mu \mathrm{M}$ TEP. The regression equation is $y=0.025 x+0.0021$, with a correlation coefficient of
Table 1 Accuracy and precision of the proposed procedure $(n=6)$

\begin{tabular}{cccc}
\hline $\begin{array}{c}\text { Amount of TEP } \\
\text { taken } / \mu \mathrm{M}\end{array}$ & $\begin{array}{c}\text { Amount of TEP } \\
\text { found } / \mu \mathrm{M}\end{array}$ & $\mathrm{RE}^{\mathrm{a}}, \%$ & $\mathrm{RSD}^{\mathrm{b}}, \%$ \\
\hline 2.50 & 2.51 & 0.4 & 1.0 \\
5.00 & 5.07 & 1.4 & 2.1 \\
10.00 & 10.18 & 1.8 & 0.3 \\
20.00 & 19.87 & -0.7 & 0.2 \\
\hline
\end{tabular}

a. Relative error.

b. Relative standard deviation.

Table 2 Analytical recovery of TEP standard added to hair samples

\begin{tabular}{lccc}
\hline & Mean $\pm \mathrm{SD} / \mu \mathrm{M}^{\mathrm{a}}$ & Recovery, \% & RSD, $\%$ \\
\hline Sample (hair) & $3.75 \pm 0.13$ & & \\
Sample + 2.5 $\mu \mathrm{M}$ TEP & $6.21 \pm 0.02$ & 98.3 & 0.32 \\
Sample + 5.0 $\mu \mathrm{M}$ TEP & $8.86 \pm 0.11$ & 102.0 & 1.24 \\
Sample + 10.0 $\mu \mathrm{M}$ TEP & $14.20 \pm 0.16$ & 105.0 & 1.13 \\
Sample + 15.0 $\mu \mathrm{M}$ TEP & $19.16 \pm 0.21$ & 102.0 & 1.03 \\
\hline
\end{tabular}

a. The values represent the mean \pm standard deviation of $n=3$.

$r=0.9999$. In order to illustrate the accuracy and precision of the proposed method, different amounts of TEP were determined, the results are listed in Table 1. To test the recovery of the proposed method, various levels of TEP were spiked with digested hair samples. As shown in Table 2, the recoveries for the TBARS analysis were all close to $100 \%$, and the average recovery of TBARS was $101.8 \pm 2.74 \%$ using the proposed method. Hence, the reliability of the proposed method was found to be acceptable.

\section{Stability of samples}

Digested hair samples were used to determine the stability of sample storage for TBARS at room temperature. The samples were stored at room temperature for twelve consecutive days and assayed in triplicate daily. As shown in Fig. 4, TBARS in the digested hair samples was stable for the first 3 days. The absorbance of TBARS significantly $(p<0.05)$ decreased at day 4 and the subsequent days when compared with day 1 , day 2 , and day 3 , respectively.

\section{Test for interference}

It has been reported that some substances interfere with the determination of the TBARS level by the TBA reaction. Because the matrices presented in blood could also be found in the hair, ${ }^{18}$ we examined the interference that resulted from substances appearing to be accumulated in the hair from blood. The criterion for interference was an absorbance intensity value varying by more than $5 \%$ from the expected value for TEP alone. It was found that albumin less than $100 \mathrm{mg} / \mathrm{ml}$, sucrose less than $12.5 \mathrm{mg} / \mathrm{ml}$, cholesterol less than $1.5 \mathrm{mg} / \mathrm{ml}$, melanin less than $1.2 \mathrm{mg} / \mathrm{ml}$, glucose less than $890 \mu \mathrm{g} / \mathrm{ml}$, and bilirubin less than $70 \mu \mathrm{g} / \mathrm{ml}$ in the mixture did not affect the estimation of the TBARS level with the proposed method.

\section{Application}

There is evidence that cigarette smoke increase the generation of lipid peroxidation. It has been indicated that reactive oxygen metabolites, and particularly the hydroxyl, may play significant roles. Smokers with coronary heart disease showed 


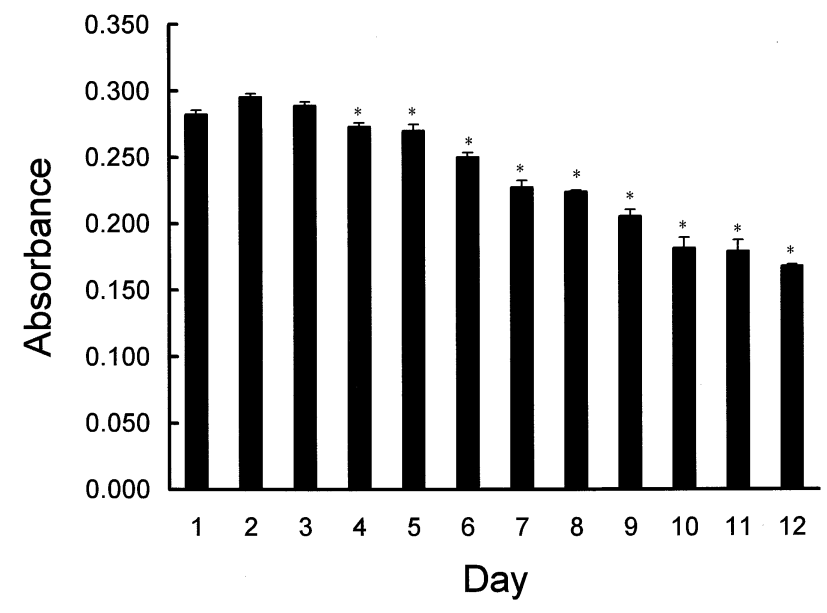

Fig. 4 Stability of a digested hair sample during storage at room temperature for 12 days. The values represent the mean \pm standard deviation of $n=3$.

$*_{p}<0.05$ compared with day 1 , day 2 , and day 3 , respectively.

significantly increased serum levels of the product of lipid peroxidation compared to non-smokers with coronary heart disease. ${ }^{27}$ The cigarette smoker serum had previously been shown to exhibit significantly less antioxidant activity than nonsmoker serum. ${ }^{28}$ In the current study, using the proposed method, TBARS in the hair of smokers and non-smokers were measured (Table 3). Because the hair conditions (such as length, gender, age, and the individual life style) may affect the difference in the content of TBARS, sixty male volunteers with the same life style and matched age were selected to evaluate the hair TBARS. The non-smoker and smoker groups were represented by 30 males ranging in age from 20 to 34 years and 30 males from 19 to 35 years of age, respectively. It was found that the mean TBARS concentration in smokers $(0.116 \mu \mathrm{mol} / \mathrm{g})$ was significantly higher than that in non-smokers $(0.096$ $\mu \mathrm{mol} / \mathrm{g})(p<0.05)$.

\section{Acknowledgements}

Financial support from the Nation Science Council of Republic of China (NSC91-2113-M-037-019) is gratefully acknowledged.

\section{References}

1. B. Halliwell and J. M. C. Gutteridge, Biochem. J., 1984, $219,1$.

2. B. Halliwell, Br. J. Exp. Pathol., 1989, 70, 737.

3. R. A. Wheatley, Trends Anal. Chem., 2000, 19, 617.

4. M. Tsuchida, T. Miura, K. Mizutani, and K. Aibara, Biochem. Biophys. Acta, 1985, 834, 196.

5. K. Akasaka, T. Takamura, H. Ohrui, H. Meguro, and K. Hashimoto, Biosci. Biotechnol. Biochem., 1996, 60, 1772.

6. D. L. Sloane, R. Leung, C. S. Craik, and E. Sigal, Nature, 1991, 354, 149.
Table 3 Statistical data of the quantitative analysis of hair TBARS in smoker and non-smoker volunteers

\begin{tabular}{lcc}
\hline & Smoker & Non-smoker \\
\hline Observations & 30 & 30 \\
Mean $(\mu \mathrm{mol} / \mathrm{g}$ hair $)$ & 0.116 & 0.096 \\
Median & 0.108 & 0.098 \\
Standard deviation & 0.030 & 0.015 \\
Standard error & 0.007 & 0.003 \\
Coefficient of variation & 27.11 & 16.06 \\
Significance (t-test) & \multicolumn{2}{c}{ Significant $(p<0.05)$} \\
\hline
\end{tabular}

7. P. G. Nouros, C. A. Georgiou, and M. G. Polissiou, Anal. Chim. Acta, 1999, 389, 239.

8. T. C. Christensen and G. Hoelmer, J. Food Sci., 1996, 61, 486.

9. M. Yasuda and S. Narita, J. Chromatogr. B, 1997, 693, 211.

10. K. J. Kunert and A. L. Tappel, Lipids, 1983, 18, 271.

11. K. N. Korizis, A. Exarchou, E. Michalopoulos, C. D. Georgakopoulos, F. Kolonitsiou, S. Mantagos, S. P. Gartaganis, and N. K. Karamanos, Biomed. Chromatogr., 2001, 15, 287.

12. H. Esterbauer, J. Lang, S. Zadravec, and T. F. Slater, Methods Enzymol., 1984, 105, 319.

13. J. W. Wong, S. E. Ebeler, R. Rivkah-Isseroff, and T. Shibamoto, Anal. Biochem., 1994, 220, 73.

14. J. Lykkesfeldt, Clin. Chem., 2001, 47, 1725.

15. K. W. Schramm, Bull. Environ. Contam. Toxicol., 1997, 59, 396.

16. P. Kintz (ed.), "Drug Testing in Hair", 1996, CRC Press, Boca Raton, New York, London, Tokyo, 293.

17. T. Uematsu, Clin. Pharmacokinet., 1993, 25, 83.

18. A. Covaci, M. Tutudaki, A. M. Tsatsakis, and P. Schepens, Chemosphere, 2002, 46, 413.

19. T. Uematsu, A. Mizuno, and K. Kosuge, "Human scalp hair as biopsy material suitable for quantitative analysis in therapeutic drug monitoring", 1995, US Department of Health, NIDA, Bethesda, 333.

20. T. Mogos, C. Panus, I. Tanase, C. Mogos, and I. Mincu, Rev. Roum. Med. Int., 1994, 32, 159.

21. K. M. Attar, A. Abdel-Aal, and P. Debayle, Clin. Chem., 1990, 36, 477.

22. F. Pragst, K. Spiegel, F. Sporkert, and M. Bohnenkamp, Forensic Sci. Internation, 2000, 107, 201.

23. T. Mogos, J. Stanesgu, I. Tanase, M. Georgescu, and I. Mincu, Rev. Roum. Med. Int., 1991, 29, 85.

24. V. Auwerter, F. Sporkert, S. Hartwig, F. Pragst, H. Vater, and A. Diefenbacher, Clin. Chem., 2001, 47, 2114.

25. Y. Hirano, K. Yamamura, K. Oguma, and K. Harada, Anal. Sci., 2001, 17, 1351.

26. A. M. Jentzsch, H. Bachmann, P. Furst, and H. K. Biesalski, Free Radic. Biol. Med., 1996, 20, 251.

27. M. A. Khan and A. Baseer, J. Pak. Med. Assoc., 2000, 50, 261.

28. P. Leanderson and C. Tagesson, Chem. Biol. Interactions, $1992,81,197$. 